\title{
Assessment of the accuracy of segmental perfusion under an occluding balloon for measuring the intrajejunal secretion of albumin and immunoglobulin $\mathrm{A}^{*}$
}

\author{
J-C RAMBAUd, $\dagger$ F DUPREY, O NOUEL, J HOSTEIN, B DELPECH, \\ AND J-J BERNIER
}

From the Research Unit on Pathophysiology of Digestion (INSERM U54), Hôpital Saint-Lazare, Paris, and Laboratory of Immunochemistry, Centre Henri Becquerel, Rouen, France

SUMMARY The suitability was investigated of the segmental perfusion technique under an occluding balloon (which prevents the contamination of the test segment by upper digestive secretions and pancreatic proteolytic enzymes) for measuring immunoglobulin A (IgA) and serum albumin outputs into the jejunal lumen. The influence of the perfusion rate and of the transintestinal water movements in the $40 \mathrm{~cm}$ long test segment was studied in 11 healthy subjects. IgA and serum albumin outputs did not differ significantly when the same isotonic solution was perfused at rates of 5 or 10 $\mathrm{ml} / \mathrm{min}$, nor when water absorption was stimulated by the presence of glucose. A possible artefact linked to intestinal wall distension by the occluding balloon was evaluated in eight subjects with complete exocrine pancreas insufficiency. IgA and serum albumin outputs obtained under the occluding balloon were compared with those found with the triple lumen non-occlusive method. Care was taken to achieve a similar flow rate and solute composition of the fluid entering the test segment with both techniques in each subject. Under these conditions, IgA and serum albumin outputs measured with both methods did not differ significantly. These findings validate the use of the segmental perfusion technique under-an occluding balloon to measure IgA and serum albumin outputs into the human small intestine.

It is well established that immunoglobulin A (IgA), mainly in its locally produced $11 \mathrm{~S}$ secretory 'form' (S-IgA), is the main type of immunoglobulin in small intestinal secretion. ${ }^{1}$ However, its secretion rate in vivo cannot be deduced from its concentration in the intraluminal fluid. Indeed, this concentration not only reflects the local production of $\operatorname{IgA}$, but also depends on at least three factors: (1) mixing of local secretion with gastric, pancreatic, biliary and overlying bowel secretions; (2) transintestinal water movements: and (3) intraluminal proteolysis, even if the secretory piece makes $\mathrm{S}-\operatorname{IgA}$ more resistant to pancreatic enzymes. ${ }^{2}$ Moreover, this last factor increases the difficulties of IgA assay.

-This study was supported by ATP grant 607892 from the Institut National de la Santé et de la Recherche Médicale, Paris, France. †Address for reprint requests: Dr J C Rambaud, INSERM U54, Hôpital Saint-Lazare, 107 bis, rue du Faubourg Saint-Denis, 75475 Paris, CEDEX 10, France.

Received for publication 29 October 1980
Serum albumin losses in the human digestive tract are usually estimated by measuring the clearance of a tracer dose of serum albumin labelled with ${ }^{51} \mathrm{Cr} .{ }^{3}$ This method probably underestimates the actual digestive losses of serum albumin, as it gives values which are close to those found for gastric losses only ${ }^{5}$ by intubation techniques.

Segmental intestinal perfusion under an occluding balloon, which prevents contamination of the test segment by upper digestive secretions, is currently the only technique allowing direct measurement of serum albumin and IgA outputs into the human bowel lumen. Such measurements would give information of the secretion rates of these substances in normal man, which are presently unknown, as well as in various pathological conditions in which the knowledge of alterations could be of pathogenic or practical interest. As the presence of the occluding balloon, as well as the flow rate and composition of 
the solution perfused, could influence the recovery of serum albumin and IgA, the aim of the present study was to evaluate the suitability of the technique for this purpose.

\section{Methods}

The study was performed in 10 healthy volunteers and in eight patients with complete exocrine pancreatic insufficiency. All gave informed consent.

INTESTINAL PERFUSION PROTOCOL

The technique of segmental perfusion of the small intestine was used to measure intrajejunal outputs of serum albumin and $\operatorname{IgA}$ in various experimental conditions.

\section{Effect of perfusion flow rate and transintestinal fluid} movements on serum albumin and IgA jejunal outputs This study was performed in 11 healthy volunteers (aged 21-35 years, 10 men and one woman) whose serum albumin levels were $38 \pm 3 \cdot 6 \mathrm{~g} / 1$. A fourlumen tube with a proximal occlusive balloon was used, according to the standard method. ${ }^{6}$ The length of the test segment was $40 \mathrm{~cm}$. The tube was swallowed by the subject before dinner and the perfusion started after overnight fasting, the infusing point being located near the duodeno-

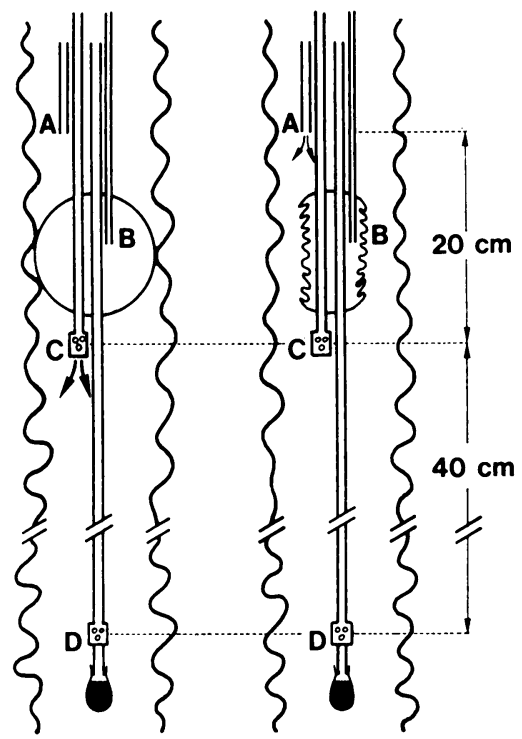

Fig. 1 Diagram of the perfusion tube used in patients with pancreatic insufficiency. Left: tube with balloon inflated. The solution is perfused in $C$ and recovered in $D$. Upper digestive secretions are aspirated in $A$, and tube $B$ allows inflation of the balloon. Right: tube with balloon deflated. The solution is perfused in $A$, and recovered in $C$ and $D$. Thus, the tube acts as a three-lumen one, the mixing segment being $A C$ and the test segment $C D$. jejunal junction under the inflated balloon (as checked fluoroscopically). Three consecutive randomised perfusion experiments were performed in all subjects: an isotonic test solution I containing per litre $115 \mathrm{mM} \mathrm{NaCl}, 10 \mathrm{mM} \mathrm{KCl}, 35 \mathrm{mM}$ mannitol, and $1 \mathrm{~g}$ polyethyleneglycol 4000 (PEG) was perfused at the rates of 5 and $10 \mathrm{ml} / \mathrm{min}$, and another solution II of identical composition, except for replacement of mannitol by an equimolar concentration of D-glucose, was perfused at the rate of $10 \mathrm{ml} / \mathrm{min}$. For each perfusion experiment four 20 minute samples were collected after a 60 minute equilibration period.

Effect of proximal occlusion of intestinal lumen on serum albumin and $\lg A$ outputs

This study was performed in eight patients with complete exocrine pancreatic insufficiency (five subjects with chronic alcoholic pancreatitis and three subjects with carcinoma of the head of the pancreas). Two subjects had a complete obstruction of the main bile duct. The perfusion tube was slightly modified as shown in Fig. 1, so that the tube could be used in the same patient, either as above for healthy subjects, the balloon being inflated, or as a triple-lumen tube. ${ }^{6}$

Each subject underwent two consecutive perfusion studies. After an overnight fast, serum albumin and IgA jejunal outputs were measured by the triple lumen technique. The test solution I was infused at the duodenojejunal junction through lumen $\mathrm{A}$ at a rate of $10 \mathrm{ml} / \mathrm{min}$; after a 60 minute equilibration period, four 20 minute proximal (lumen C) and distal (lumen D) samples were collected. Proximal sampling was carried out by gentle aspiration with a syringe at the constant rate of $2 \mathrm{ml} / \mathrm{min}$ and distal collection was made by free siphonage. The flow rate and composition of the fluid entering the test segment were immediately determined by measuring the concentration of PEG in the test solution and the osmolality and the concentrations of PEG and electrolytes in the proximal samples. Thereafter, during the afternoon, serum albumin and IgA outputs were determined at the same jejunal site, using the proximal occlusive balloon technique. In each subject, the electrolyte and mannitol composition and the rate of infusion in lumen $\mathrm{C}$ of the test solution mimicked as closely as possible those of the fluid entering the test segment during the preceding triple lumen tube perfusion. Four 20 minute samples were taken through lumen $\mathrm{D}$ by siphonage after a 60 minute equilibration period. Thus, in each subject, the site and length of the test segment CD, the osmolality, electrolyte composition, and flow rate of the perfused solution were the same during the two perfusions, although the intestinal lumen 
was occluded in the first, but not in the second. Patients continued to fast during the whole study, and received an intravenous infusion of glucose and balanced electrolyte solution (with insulin when necessary).

All perfusate samples obtained in normal subjects and patients were recovered at $0^{\circ} \mathrm{C}$. Diisopropylfluorophosphate (DFP), a potent proteolysis inhibitor, was added to an aliquot fraction which was homogenised and stored at $-20^{\circ} \mathrm{C}$ until serum albumin and IgA were measured.

\section{ANALYTICAL METHODS}

Serum albumin and IgA concentrations were measured without concentration by the radial immunodiffusion technique ${ }^{7}$ using antisera to human serum albumin (Behringwerke, Marburg, FAR) and to alpha chain (Institut Pasteur, Paris) and standardised human serum as the standard scale. Trypsin and chymotrypsin activities were assayed by the method of Figarella et $a l^{8}{ }^{8}$ and PEG by that of Hyden. ${ }^{9}$ Sodium and potassium were measured by flame photometry; chloride and bicarbonate were analysed on a Technicon autoanalyser. The concentration of mannitol in the proximal samples obtained during the triple lumen tube experiments was calculated from the difference between the actual osmolalities measured with a Fisk osmometer and the osmolality calculated from electrolyte concentrations of the perfusate.

CALCULATION AND EXPRESSION OF RESULTS

The net movements of water, electrolytes, serum albumin and IgA in the test segment, and the flow rate of fluid entering the test segment in the triple lumen experiments were calculated from concentrations of PEG, electrolytes, serum albumin and IgA according to standard methods. ${ }^{6}$ All results are expressed as mean $\pm \mathbf{S E M}$; positive and negative values indicate secretion and absorption respectively. Statistical comparisons were performed according to the paired $t$ test for studies in pancreatic insufficiency. In normal subjects, in whom three variables were studied, variance analysis was made by the block method and statistical comparisons were performed by the Newmann-Keuls test.

\section{Results}

No detectable trypsin and chymotrysin activity was found in all intestinal perfusates, either in healthy subjects or in patients with pancreatic insufficiency. Moreover, the diffusion rings obtained during the determination of serum albumin and IgA concentration in the perfusates had very distinct external limits.

Figs. 2 and 3 show that outputs of serum albumin

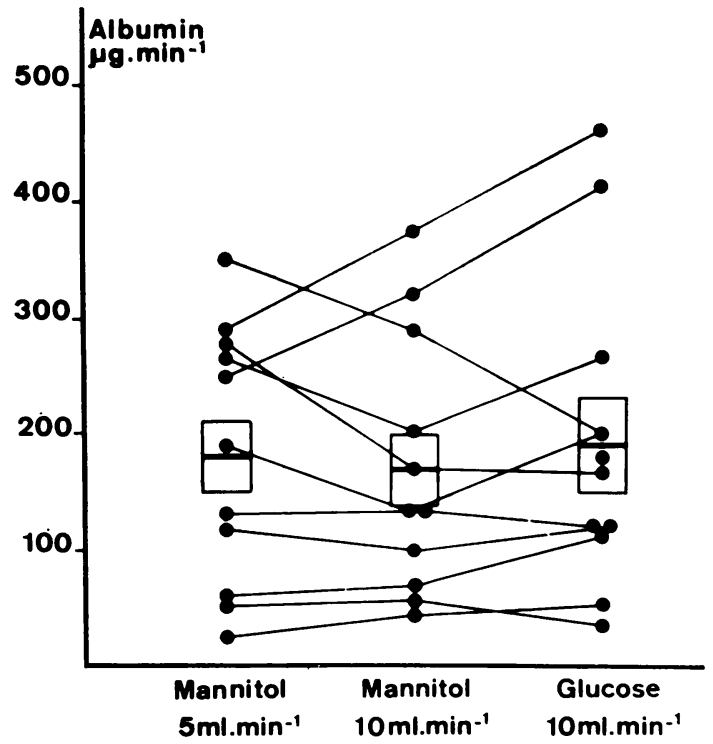

Fig. 2 Jejunal outputs of serum albumin during perfusion of the mannitol-containing solution at two flow rates and during perfusion of the glucose-containing solution. These outputs do not differ significantly. Bars indicate mean $\pm S E M$.

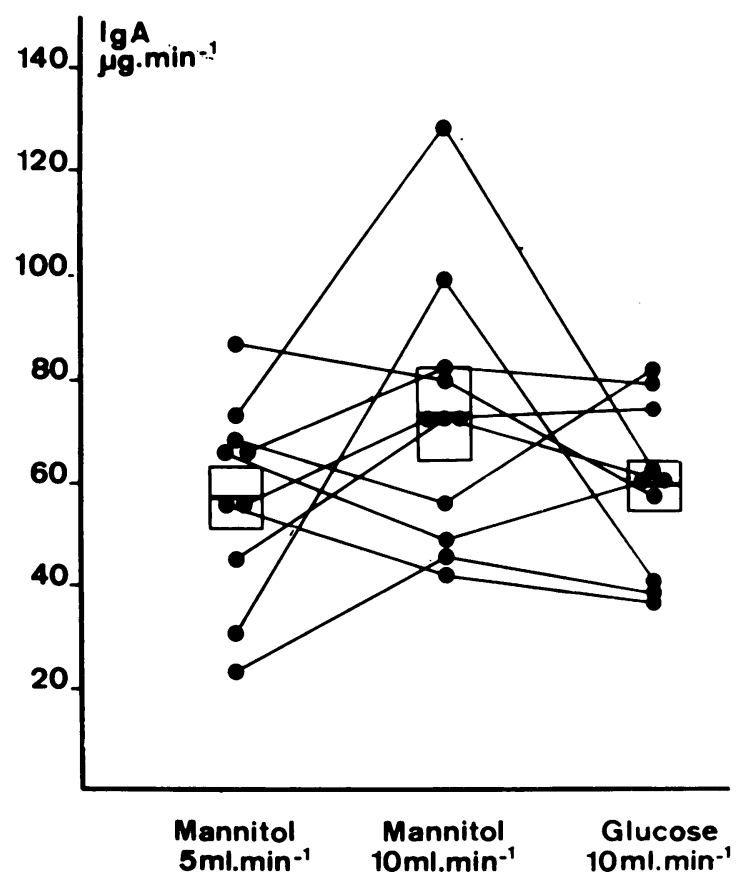

Fig. 3 Jejunal outputs of $\operatorname{IgA}$ during perfusion of the mannitol-containing solution at two flow rates and during perfusion of the glucose-containing solution. These outputs do not differ significantly. Bars indicate mean $\pm S E M$. 
and IgA were not statistically different during perfusions performed in healthy subjects at $5 \mathrm{ml}$ and $10 \mathrm{ml} / \mathrm{min}$; nor when glucose was substituted for mannitol in the test solution. Transintestinal water movements per minute were $-0.2 \pm 0.1 \mathrm{ml}$ for $5 \mathrm{ml}$ mannitol perfusions, $0.1 \pm 0.2 \mathrm{ml} / \mathrm{min}$ for $10 \mathrm{ml}$ mannitol perfusions, and $-3.3 \pm 0.2 \mathrm{ml}$ for $10 \mathrm{ml}$ glucose perfusion. The difference between the last two figures was statistically significant $(P<0.01)$, whereas that observed between the first two figures was not significant. Jejunal serum albumin clearance (intestinal serum albumin output: blood albumin level) in normal subjects, for all types of perfusions taken together was $6 \cdot 26 \pm 0 \cdot 72(\mathrm{n}=33) \mathrm{ml} / 24 \mathrm{~h} / 40 \mathrm{~cm}$ of gut.

In patients with pancreatic insufficiency, serum albumin and IgA outputs per minute (Fig. 4) were $66 \pm 10(n=7)$ and $70 \pm 14(n=7) \mu \mathrm{g}$ respectively during triple lumen tube experiments and $90 \pm 18$ $(\mathrm{n}=7)$ and $67 \pm 18 \quad(\mathrm{n}=7) \mu \mathrm{g}$ respectively during perfusions under the occlusive balloon, the differences observed for these two substances between the two perfusion techniques not being statistically significant. There were also no significant differences

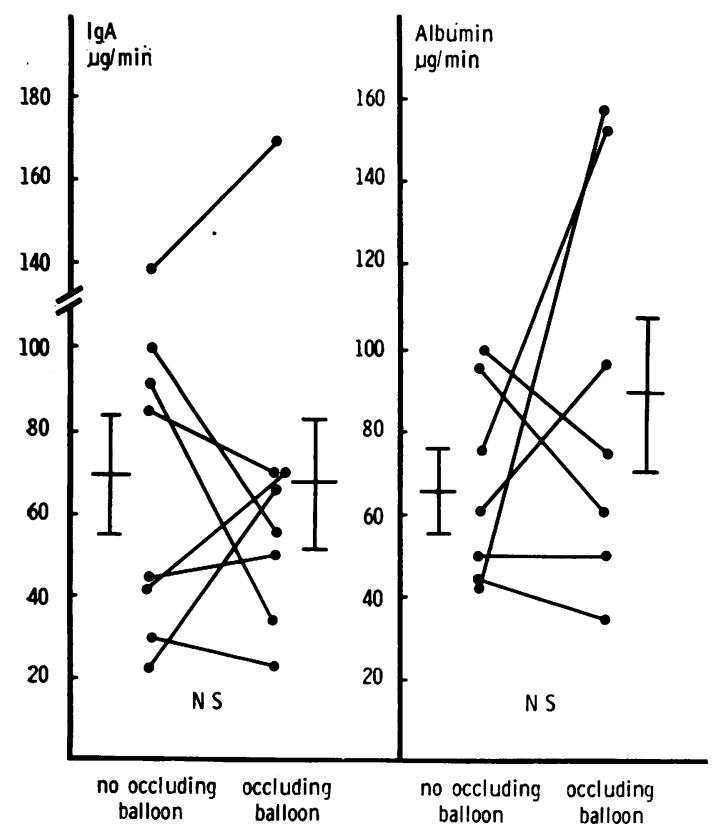

Fig. 4 Jejunal outputs of $\operatorname{Ig} A$ and albumin in patients with complete exocrine pancreatic insufficiency. Outputs found with the triple lumen tube (no occluding balloon) do not differ significantly from those obtained with the perfusion under an occluding balloon. Bars indicate means $\pm S E M$. between patients with or without obstruction of the main bile duct.

\section{Discussion}

Occlusion of the intestinal lumen above the test segment in order to prevent the contamination of the perfusate by pancreatic enzymes is necessary and sufficient $^{10}$ to avoid significant intraluminal proteolysis. However, the presence of this occlusive balloon could affect the local or regional blood and lymphatic circulation, and influence the intraluminal secretion of circulating polypeptides, such as serum albumin and $7 \mathrm{~S} \mathrm{IgA.} \mathrm{This} \mathrm{possible} \mathrm{artefact,} \mathrm{as} \mathrm{well} \mathrm{as} \mathrm{that}$ associated with the absence of bile salts in the perfusate, does not seem important, as shown the similarity between serum albumin and IgA outputs measured by the occlusive balloon method and by the triple lumen tube technique in patients with complete exocrine pancreas insufficiency who do or do not have obstruction of the main bile duct.

IgA and serum albumin can stick to the intestinal mucosa, and other factors such as the flow rate of the perfusion, as well as transintestinal fluid movements, could influence the recovery of these polypeptides in the perfusate. In fact, neither the doubling of the perfusion rate nor the stimulation of water absorption by glucose had a significant effect on serum albumin and IgA outputs. We have also shown by these experiments that serum albumin and IgA secretion rates (in the absence of antigenic stimulation) were steady over a seven-hour perfusion period.

Assuming that the mean length of the whole small intestine intubated by the four lumen tube with an occlusive balloon is $160 \mathrm{~cm}$ (unpublished data) and that the secretion rate of serum albumin remains constant from the pylorus to the ileocaecal junction, the mean small intestinal clearance of serum albumin in the 10 healthy subjects we have studied would be $25 \mathrm{ml} /$ day. This figure is of the same order of magnitude as that found for gastric serum albumin clearance determined by direct recovery of gastric juice. ${ }^{45}$ The sum of these two figures is nearly double the total tract clearance of serum albumin determined by Waldman's method ${ }^{3}$, which is thus probably an underestimate. Apart from its physiological interest, measurement of serum albumin losses in various parts of the intestine would allow us to determine the precise site of plasma protein losses localised in a segment of the digestive tract; this would permit a surgical resection if the normal medical treatment failed.

Radial immunodiffusion with a human serum standard is not an accurate method for a precise evaluation of $\operatorname{IgA}$ concentrations in intestinal juice. 
Indeed, it underestimates the 11S S-IgA, by at least a factor of two. ${ }^{11}$ Thus, the values of IgA secretion rates reported here were purely conventional and were used only to compare in the same subjects IgA outputs under various experimental conditions. Provided an accurate measurement of S-IgA is available, the method of segmental perfusion under an occluding balloon would allow the determination of the intraintestinal output of S-IgA in the normal subject, which is at present unknown, as well as in pathological conditions (such as coaeliac disease, Crohn's disease, primary immunodeficiency syndromes, cirrhosis of the liver) where abnormal S-IgA secretion could be of pathogenic interest.

We acknowledge the technical assistance of Mrs M Bovet, C Franchisseur, M Maurel, and M Rongier.

\section{References}

'Tomasi TB. Secretory immunoglobulins. $N$ Engl $J$ Med 1972; 287: 500-6.

${ }^{2}$ Lindh E. Increased resistance of immunoglobulin A dimers to proteolytic degradation after binding of secretory component. $J$ Immunol 1975; 114: 284-6.

${ }^{3}$ Waldman TA, Wochner RD, Strober W. The role of the gastrointestinal tract in plasma protein metabolism, studies with ${ }^{51} \mathrm{Cr}$ albumin. Am J Med 1969; 46: 275-85. 'Vidon N, Bernier JJ. Détermination par la sérumalbumine marquée au ${ }^{51} \mathrm{Cr}$ des pertes d'albumine plasmatique dans l'estomac chez l'homme. Biol Gastroenterol 1971; 2: 153-68.

${ }^{5}$ Brassine A. Gastric clearance of serum albumin in normal man and in certain gastroduodenal disorders Gut 1974; 15: 194-9.

-Modigliani R, Rambaud JC, Bernier JJ. The method of intraluminal perfusion of the human small intestine. I-Principle and technique. Digestion 1973; 9: 176-92.

${ }^{7}$ Mancini C, Carbonara AO, Heremans JF. Immunochemical quantification of antigens by simple radial immunodiffusion. Immunochemistry 1965; 2: 235-54.

${ }^{8}$ Figarella C, Taulier J, Sarles H. Dosage de la chymotrypsine et de la trypsine dans le suc duodénal. Bull Soc Chim Biol 1965; 47: 679-86.

${ }^{\circ}$ Hyden S. A turbidimetric method for the determination of the higher polyethylene glycols in biological materials Ann Roy Agr Coll 1955; 22: 139-45.

${ }^{10}$ Walker WA, Wu M, Isselbacher KJ, Bloch KJ. Intestine uptake of macromolecules. IV-The effect of pancreatic duct ligation on the breakdown of antigen and antigenantibody complexes on the intestinal surface. Gastroenterology 1975; 69: 1223-9.

"Bull DM, Bienenstock J, Tomasi TN. Studies on human intestinal immunoglobulin A. Gastroenterology 1971; 60: $370-80$. 